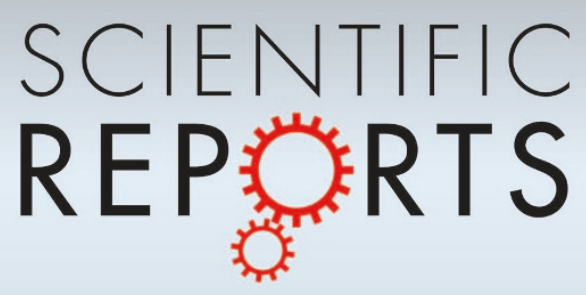

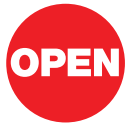

SUBJECT AREAS:

MAGNETIC RESONANCE

IMAGING

CELLULAR MOTILITY

EXTRACELLULAR MATRIX

IMAGE PROCESSING

Received

11 October 2012

Accepted

1 May 2013

Published

23 May 2013

Correspondence and requests for materials should be addressed to

A.P.K. (KoretskyA@ ninds.nih.gov)

* These authors contributed equally to this work.

\section{Measuring collective cell movement and extracellular matrix interactions using magnetic resonance imaging}

\author{
Yun Chen' ${ }^{1}$ Stephen J. Dodd ${ }^{1 *}$, Michael A. Tangrea ${ }^{2 *}$, Michael R. Emmert-Buck² \& Alan P. Koretsky'
}

\footnotetext{
${ }^{1}$ National Institutes of Neurological Disorders and Stroke, National Institutes of Health, ${ }^{2}$ National Cancer Institute, National Institutes of Health.
}

Collective cell behaviors in migration and force generation were studied at the mesoscopic-level using cells grown in a 3D extracellular matrix (ECM) simulating tissues. Magnetic resonance imaging (MRI) was applied to investigate dynamic cell mechanics at this level. MDCK, NBT2, and MEF cells were embedded in 3D ECM, forming clusters that then migrated and generated forces affecting the ECM. The cells demonstrated MRI contrast due to iron accumulation in the clusters. Timelapse-MRI enabled the measurement of dynamic stress fields generated by the cells, as well as simultaneous monitoring of the cell distribution and ECM deformation/remodeling. We found cell clusters embedded in the 3D ECM can exert translational forces to pull and push, as well as torque, their surroundings. We also observed that the sum of forces generated by multiple cell clusters may result in macroscopic deformation. In summary, MRI can be used to image cell-ECM interactions mesoscopically.

ollective cell migration is defined as an orchestrated movement among interconnected cell groups and is required for normal tissue development ${ }^{1}$. Pathologically, collective cell migration is exploited by cancer cells as an efficient invasion strategy that can be modeled in the laboratory ${ }^{2}$. For example, ex vivo melanoma explants cultured in a 3D collagen gel demonstrate invasive migration in multicellular clusters ${ }^{3,4}$. In both normal and pathological states, collective migration is a mechanical force-dependent process whereby aggregated cells generate traction forces through actin-myosin contraction and move forward against tensile forces distributed along cell-cell adhesive contacts ${ }^{5}$. The traction forces drive ECM remodeling surrounding the cells, resulting in a topological rearrangement of ECM fibers that in turn shape the tissue microenvironment $t^{6,7}$, or promote metastatic phenotypes ${ }^{7,8}$. To date, the field of cell mechanics has mostly focused on the migrating behaviors of single cells at a microscopic level and studies characterizing cell behaviors in a more physiologically relevant $3 \mathrm{D}$ culture system have advanced only recently $y^{9,10}$. In order to better understand physiology at the tissue level, there have been emerging interests in the study of mesoscopic biological phenomena ${ }^{11-13}$. Thus, knowledge of how aggregated cells move in concerted ways to interact with their 3D environment needs to be comprehensively analyzed. Such an understanding would provide important insight into the mechanisms of many physiological and pathological processes, including embryonic development, cancer invasion, organ tubulogenesis, and angiogenesis.

The goal of our study was to develop a platform to systematically investigate collective cell migration and associated force generation which shapes tissue structures in 3D, physiological conditions. There are many important biological processes involving simultaneous cell migration and dynamic force generation at the mesoscopic level. One of the most well documented examples is embryonic development where migrating cells reshape the embryo through defined deformation such as gastrulation and invagination. Another example is during metastasis, where cancer cells and their altered tissue form a new and dynamic "organ-like tissue", which deforms the surrounding stroma as the malignancy progresses ${ }^{14}$. To investigate such processes require the capacity to quantitatively image objects at mesoscopic scales, encompassing both a millimeter-range field of view with micron resolution. While optical microscopy is a powerful tool in the realm of sub-micron scales, it has critical limitations in achieving 3D imaging at the mesoscopic level, particularly regarding the size of the field of view. Due to the physical nature of modern microscope optics, imaging at a millimeter-field of view requires a complex rastering process that is time-consuming, despite continuous improvements in beam scanning technology and signal generation rates ${ }^{15-17}$. A second limitation is the restricted field depth of optical microscopy where samples thicker than $0.5 \mathrm{~mm}$ usually present challenges for visualization. In addition, it has been shown that cells 
respond to the rigidity of an underlying glass coverslip beneath a thin gel $^{18}$. Thus imaging thin gels can result in misleading conclusions about cell behaviors embedded in a soft ECM. In contrast, MRI has intrinsic 3D capacity for imaging samples at sub-millimeter resolution and thus may provide an alternative for analyzing collective cell mechanics.

There is a large body of literature demonstrating that MRI contrast agents can be successfully employed to label cells for transplantation studies in vivo ${ }^{19-23}$ and track individual cells if enough contrast is provided $^{24}$. Other recent studies have shown the feasibility of observing cell clusters mesoscopically using $\mathrm{MRI}^{25,26}$. In the present work, we extend this line of inquiry and evaluate the use of MRI as a tool to monitor dynamic behaviors of collective cell migration. Furthermore, by imaging the deformation of the ECM, we examined if the stress fields associated with the collective movement could be derived. Though 3D ECM deformation/remodeling has been qualitatively characterized in previous studies by optical imaging approaches ${ }^{27-29}$, our goal was to develop a system that enables characterization of ECM deformation/remodeling dynamically and quantitatively while simultaneously tracking collective cell migration.

\section{Results}

MRI detects cell clusters in 3D ECM. The typical spatial resolution limit of current commercial small animal MRI technology is approximately $(20-50 \mu \mathrm{m})^{3} /$ voxel. Although it is still a challenge to image a single unlabeled cell with MRI, it is possible to visualize small cell aggregates such as epithelial cell clusters. MDCK cells of epithelia origin first served as an in vitro model to assess if MRI can be used to monitor collective cell behavior of clusters. A dualmodality experimental system was built so that the MRI findings could be compared to optical microscopy. Briefly, MDCK cells were transfected with GFP for fluorescent imaging and seeded in a relatively thin $(0.5 \mathrm{~mm}) 3 \mathrm{D}$ collagen gel matrix (diameter $=15 \mathrm{~mm}$, thickness $=0.5 \mathrm{~mm}$ ) to minimize light scattering and photon penetration issues, and then MRI was performed by a $11.7 \mathrm{~T}$ scanner. Four hours after seeding, contrast could be detected by spin echo sequence $\left(\mathrm{TE} / \mathrm{TR}=5 \mathrm{~ms} / 30 \mathrm{~ms}\right.$, flip angle $=15^{\circ}$ ) (figure 1a). As a control, MDCK cells without GFP transfection were also scanned following the same protocol and similar MRI contrast was detected indicating that GFP was not the cause for the observed contrast (data not shown).

The observed contrast was due to shortening of $\mathrm{T}_{2}{ }^{*}$, resulting in the darker regions in the MRI image (Figure 1). To verify these areas were in fact MDCK cells, the collagen gel was examined by optical microscopy. At low magnification $(10 \times)$, fluorescent spots were observed that corresponded to the locations with dark contrast (figure 1b). Closer examination of the fluorescent spots at higher magnification $(40 \times)$ revealed aggregated cell clusters (figure 1c). To date, we have been able to image clusters containing as few as 30 to 40 unlabeled cells; however, the minimal cell number necessary to create an MRI-detectable cluster was not determined.

When aggregated, MDCK cells exhibited a highly orchestrated collective behavior with a synergistic effect on migration and force generation $^{30-32}$. We were able to track the motility of MDCK cell clusters by timelapse MRI (figure $1 \mathrm{~d}$, movie $\mathrm{S} 1$ ). 3D real-time images of moving MDCK clusters were recorded and the trajectory of single clusters were calculated for the displacement in consecutive frames. The average speed of the 12 cell clusters tracked was $0.39 \pm 0.37 \mu \mathrm{m} /$ minute (figure 1e), consistent with previous observations of collectively migrating epithelial cells ${ }^{33,34}$, demonstrating that MRI can dynamically track clusters of unlabeled cells for an extended time period (30 hours).
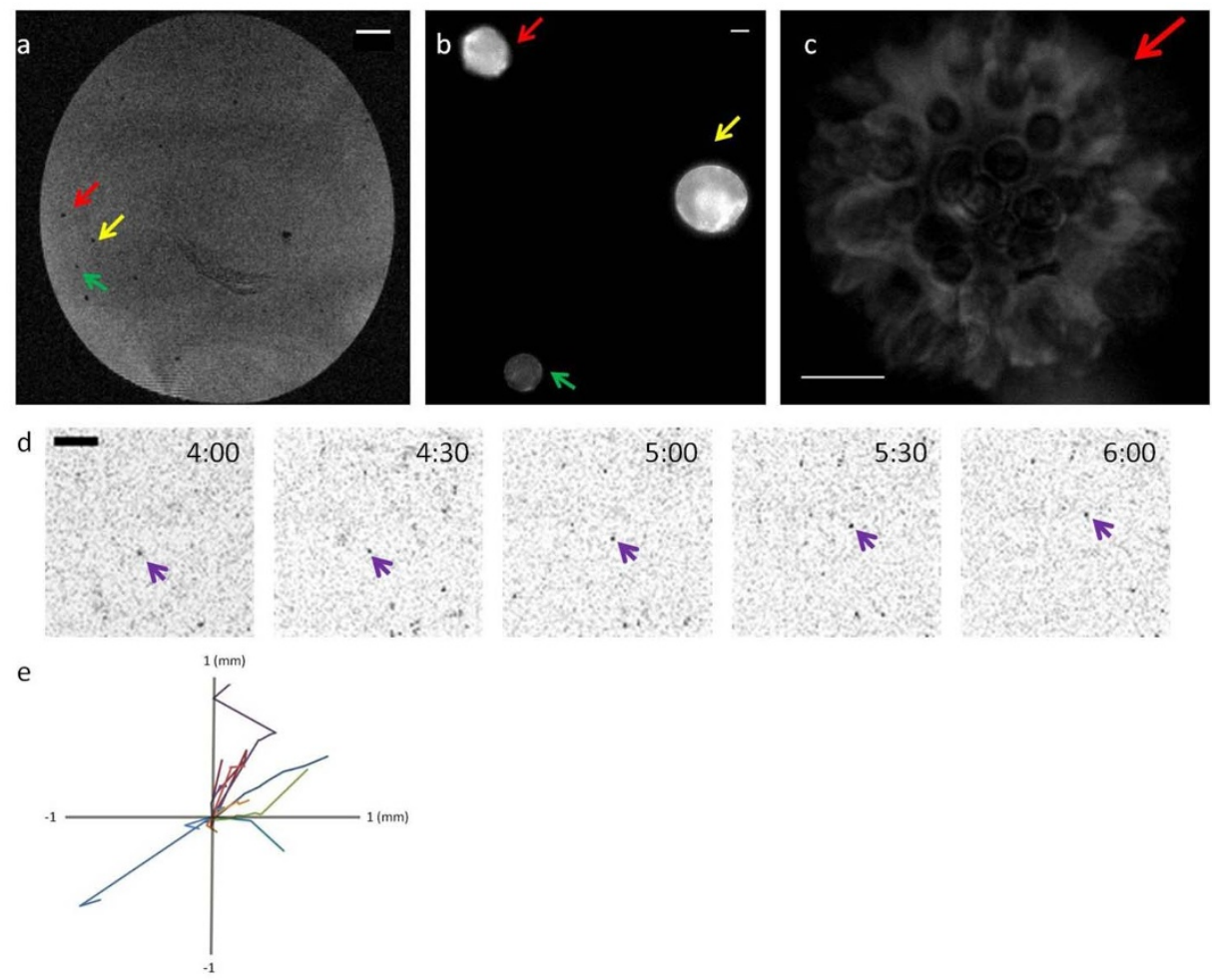

Figure 1 MRI Detects Cell Clusters in 3D ECM. MDCK cells (dark objects) observed by MRI and highlighted by green, yellow and red arrows (a) correspond to fluorescent spots observed via epifluorescent microscopy indicated by green, yellow and red arrows (b). A higher magnification image of the red arrow cell cluster (b) reveals that multiple cells are clustered in the spot (c). The cell clusters could also be detected dynamically from 4-6 hours after seeding. A moving cluster is marked with the purple arrow throughout acquisition time. (d). Multiple clusters were tracked and their trajectories are shown in (e). The time stamps shown in each image indicate the time when the image was taken after cell encapsulation.

Scale bars: (a) $1 \mathrm{~mm}$; (b) $10 \mu \mathrm{m}$; (c) $10 \mu \mathrm{m}$; (d) $1 \mathrm{~mm}$. 
Clustering and iron accumulation in MDCK cells contribute to MRI contrast. The spin echo sequence used in this study was designed to detect differences in traverse relaxation time, namely $\mathrm{T}_{2}{ }^{*}$, between different substances. The observation that MDCK cells were first detected in MRI after being seeded into the 3D collagen ECM for four hours led us to speculate they underwent a structural change that affected their traverse relaxation time, resulting in the contrast between the cells and the surrounding environment. Measuring the $\mathrm{T}_{2}$ and $\mathrm{T}_{2}{ }^{*}$ relaxation time of cells at one, four, and eight hours after seeding showed that these values decreased significantly over the first four hours: from $100.2 \mathrm{~ms}$ to $83.5 \mathrm{~ms}$ for $\mathrm{T}_{2}$ at four hours, and from $63.1 \mathrm{~ms}$ to $14.1 \mathrm{~ms}$ at four hours for $\mathrm{T}_{2} *$ (Figure $2 \mathrm{~d}, \mathrm{e}$ ). The values of $\mathrm{T}_{2}$ and $\mathrm{T}_{2} *$ relaxation time stayed at the ranges of $80 \mathrm{~ms}$ and $15 \mathrm{~ms}$ without further decrease at eight hours (Figure 2d, e).

There are many known factors that affect traverse relaxation and subsequently contribute to image contrast in MRI, including local iron density variations ${ }^{35,36}$, as well as differences in diffusion of water molecules due to structural hindrances ${ }^{37-40}$. The time when cells were detected by MRI coincided with the time when cell clustering was observed by optical microscopy; it is thus possible that structural rearrangement and physiological adaptation occurred among the cells, leading to iron accumulation in the clusters, as well as slower, confined water diffusion. As a result, a change in $\mathrm{T}_{2}{ }^{*}$ and the subsequent contrast was observed.

To examine the mechanism behind the observed changes in $\mathrm{T}_{2} *$ contrast in the cell-embedded 3D ECM, the collagen gel was fixed at one, two, four, and eight hour(s) after cell seeding and subsequently cut into $5 \mu \mathrm{m}$-thick histological sections along the $\mathrm{z}$-axis. The slides were then stained by hematoxylin and eosin (H\&E) to assess cell morphology. Microscopically, we found that individual cells began to aggregate at two hours after seeding. At four hours most of the cells were in contact with other cells and formed clusters across the 3D ECM (figure 1a). Additionally, the slides were analyzed by iron staining and higher iron content was found in the cell clusters at four hours after seeding (figure 2b). The high local iron density in the cell clusters likely contributed to the shortening of $\mathrm{T}_{2}$ and $\mathrm{T}_{2}{ }^{*}$ relaxation (figure $2 \mathrm{~d}, \mathrm{e}$ ) and the subsequent image contrast observed at this time point.

To further verify that the $\mathrm{T}_{2} *$ contrast was associated with cell clustering; pre-formed clusters were embedded in the 3D matrix and treated with hepatocyte growth factor (HGF). HGF is known to cause disassembly of epithelial cell-cell junctions ${ }^{41-43}$ and it has previously been shown that MDCK cells express the HGF receptor and exhibit strong scattering activity upon HGF stimulation ${ }^{42,43}$. Timelapse MRI revealed that the contrast gradually disappeared over four hours after HGF treatment (figure 2c), indicating that disassembly of MDCK cell clusters caused a loss in $\mathrm{T}_{2}{ }^{*}$ contrast.

Large-scaled ECM deformation and remodeling tracked by MRI. The mechanical interactions between aggregated cells and the surrounding ECM environment contributes to ECM deformation and remodeling ${ }^{44}$, and $3 \mathrm{D}$ matrix deformation assays have been applied as a qualitative method to assess traction forces generated a
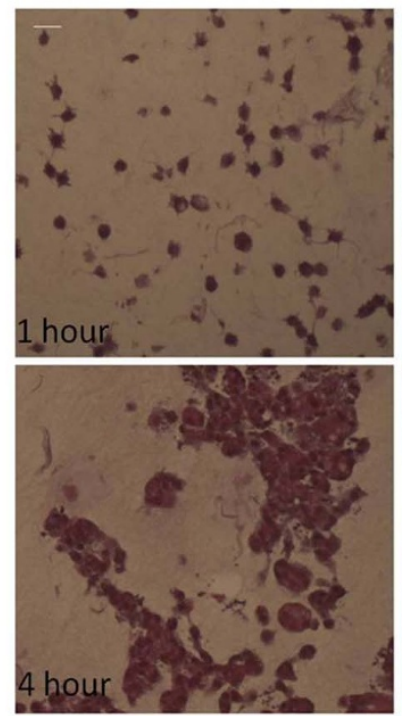

b

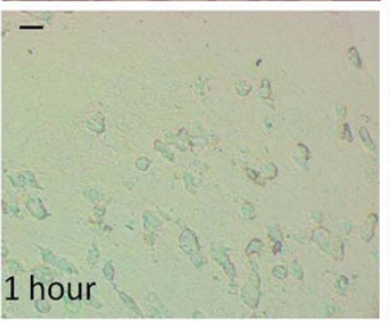

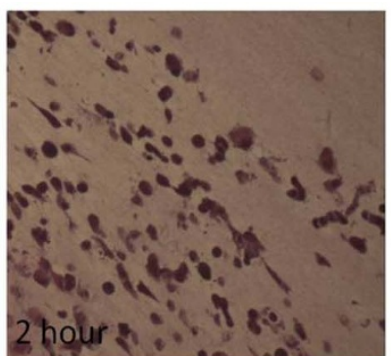
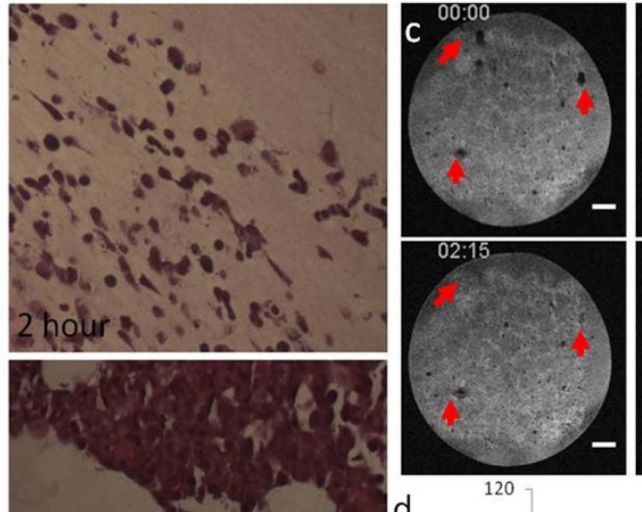

d

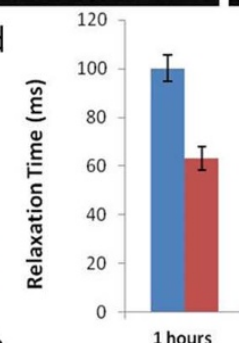

e
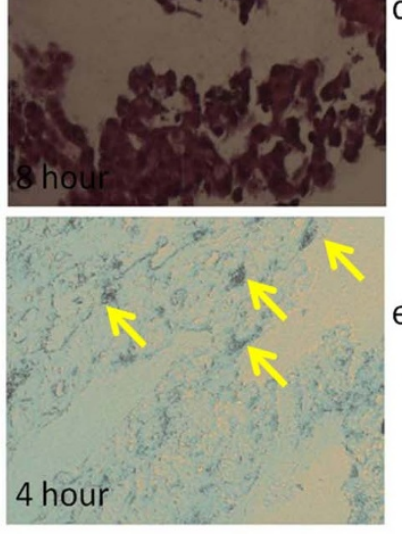

1 hours
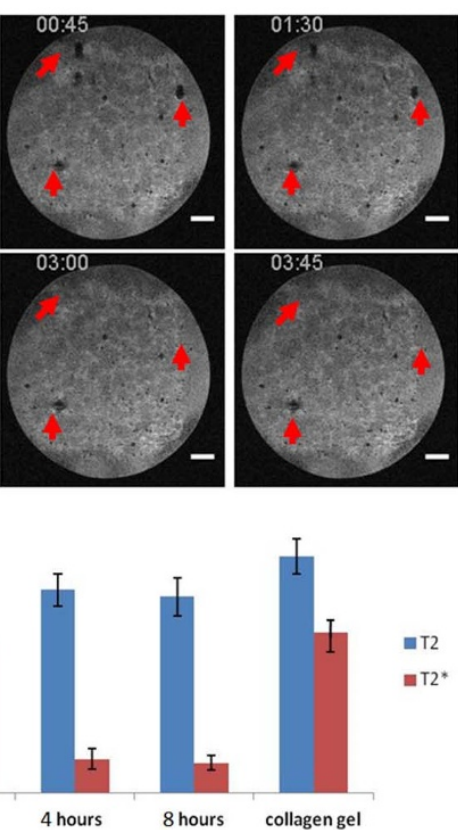

$\square \mathrm{T} 2$

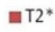

\begin{tabular}{|l|r|r|r|r|}
\hline & \multicolumn{1}{|c|}{ 1 hours } & \multicolumn{1}{l|}{ 4hours } & 8hours & collagengel \\
\hline$T 2$ & $100.2 \pm 5.4$ & $83.5 \pm 6.5$ & $80.8 \pm 7.8$ & $97.3 \pm 7.3$ \\
\hline$T 2 *$ & $63.1 \pm 4.7$ & $14.1 \pm 4.2$ & $12.5 \pm 3.1$ & $65.9 \pm 8.1$ \\
\hline
\end{tabular}

Figure $2 \mid$ Cell clustering and iron accumulation contributes to MRI contrast. The cell-embedded collagen gel was fixed at one, two, four, and eight hour(s) after cells were seeded, and then cut into $5 \mu \mathrm{m}$-thick slices along the $\mathrm{z}$-axis and stained by hematoxylin and eosin (H\&E). Embedded cells exhibit an evolving morphology at different time points, gradually becoming clusters (a). Individual cells started to aggregate at 2 hours after seeding; and at 4 hours after seeding most of the cells were in contact with other cells, forming clusters across the 3D matrix. Iron staining showed iron accumulation in the cell clusters (b, indicated by yellow arrows). Cells treated by HGF, a known scattering factor for MDCK cells, lost MRI contrast after 4 hours of treatment. The cell clusters are indicated by red arrows (c). The images shown in (c) are the z-projection of the whole specimen. The clustering of embedded MDCK cells coincide with the decrease of $\mathrm{T}_{2}$ and $\mathrm{T}_{2}{ }^{*}$ relaxation times $(\mathrm{d}, \mathrm{e})$. The time stamps shown in each image indicate the time the image was taken after cell encapsulation. Scale bars: (a) $10 \mu \mathrm{m}$; (b) $10 \mu \mathrm{m}$; (c) $1 \mathrm{~mm}$. 
by cells ${ }^{28,29,45}$. Conventionally, ECM deformation/remodeling is evaluated by the surface contour changes of a disc-shaped, cell-embedded 3D matrix at different time points ${ }^{28,45}$. However this conventional method overlooks the anisotropic deformation throughout the volume and does not provide information about the dynamic distribution of embedded cells inside the ECM. Therefore, to simultaneously monitor cell migration and ECM deformation/remodeling quantitatively, $8 \times 10^{7} \mathrm{MDCK}$ cells were embedded in a ring-shaped collagen gel (inner diameter: $5.5 \mathrm{~mm}$, outer diameter: $9 \mathrm{~mm}$; figure $3 \mathrm{a}, \mathrm{b}$ ) and then imaged for 30 hours. The ECM gel was molded into the shape of a ring so the deformation could be easily detected by visual inspection (figure 3a, b; movie S2).

To evaluate the dynamic deformation exerted by the embedded MDCK cells, the 3D surface contour of the ring-shaped collagen gel at each acquisition time was fitted into a triangular mesh of 1340 nodes and 5783 elements using the Delaunay tessellation method. The displacement of each node between consecutive acquisition times was then computed to estimate the deformation. Each element was assigned isotropic linear elastic material properties with elastic modulus $\sim 15 \mathrm{kPa}$ based on previous estimations $\mathrm{s}^{7,46,47}$. The mechanical stress of each element, which resulted from forces exerted by the cell cluster onto its surrounding environment, was then calculated as a product of the matrix stiffness with the displacement components using the following formulas ${ }^{48}$ :

$$
\begin{gathered}
\varepsilon=\frac{1}{2}\left[(\nabla \mathrm{u})^{T}+(\nabla \mathrm{u})\right] \\
\sigma=\mathrm{D} \bullet \varepsilon
\end{gathered}
$$

Where $\mathrm{u}$ represents the displacement vector, $\varepsilon$ represents the strain, $\sigma$ represents the mechanical stress experienced by the element, and D represents the matrix stiffness.

The calculation showed that the stress ranged from 0 to $30 \mathrm{nN} /$ $\mu \mathrm{m}^{2}$, exhibiting heterogeneous deformation throughout the gel (figure 3e, movie S3). The amplitude of the deformation varied both temporally and spatially.

To investigate if there was a correlation between dynamic cell distribution and ECM deformation/remodeling, cell clusters and the surface contour of the matrix were separately segmented, color coded (figure 3c), and reconstructed back into the 3D time series images (figure 3d, movie S4), where the distribution of cell clusters and matrix deformation could be visualized simultaneously in red
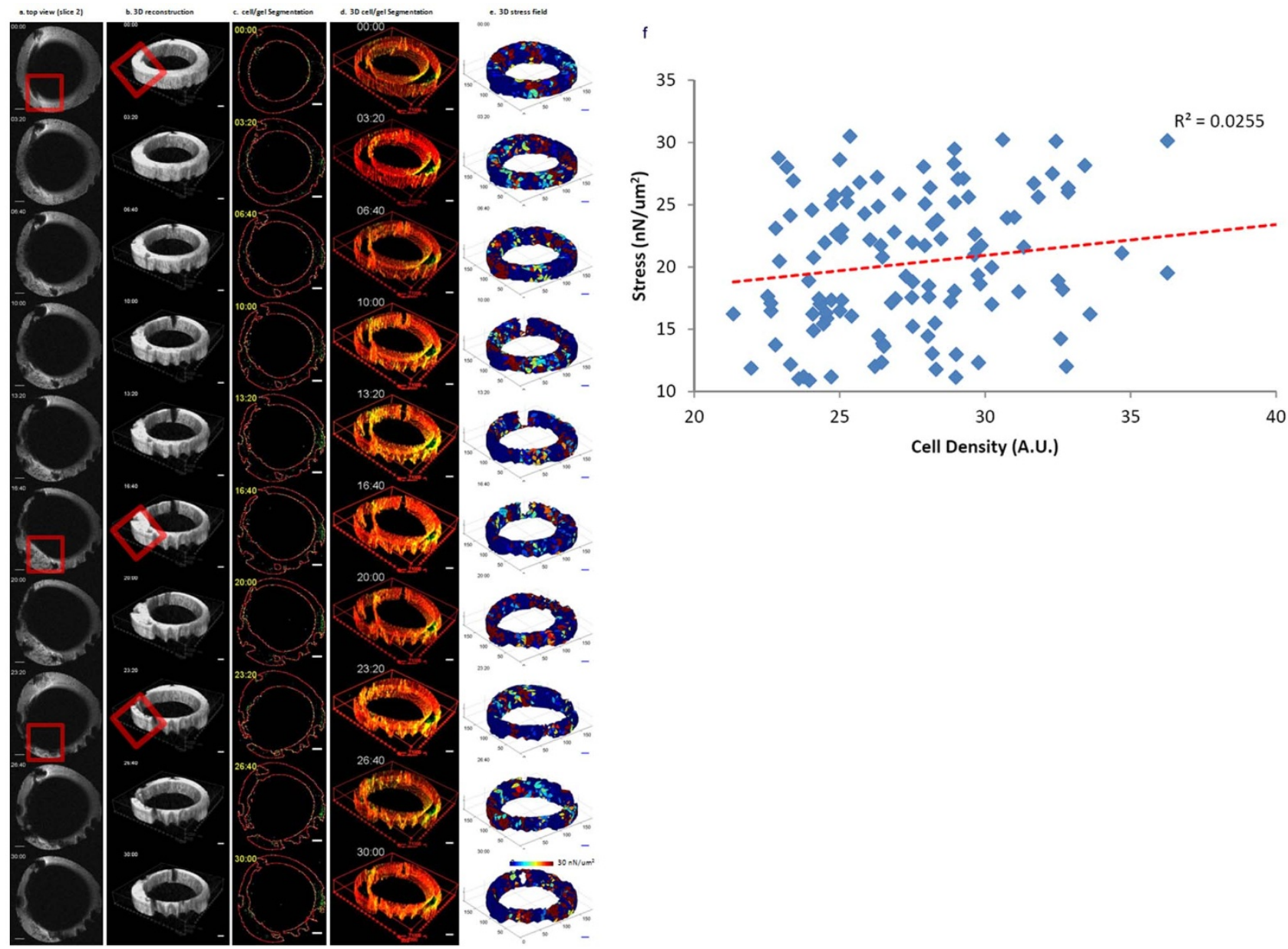

Figure $3 \mid$ MRI tracks large-scaled ECM deformation and remodeling. The ring-shaped, cell-embedded collagen gel was deformed over 30 hours of the imaging period. The deformation in the second slice of 3D stack ( 32 slices in total) is shown in (a), where the red boxes highlight the area undergoing visually detectable shape changes over time. The 3D reconstruction of the ECM gel is shown in (b), where the red boxes highlight the corresponding area marked in (a). Cell clusters and the surface contour of the collagen gel were separately segmented and color-coded in green and red, respectively. Panel (c) shows a segmented 2D slice of the 3D image. The segmented images were then reconstructed back into 3D time series images (d), where the cell cluster distribution and matrix deformation can be visualized simultaneously. The 3D images were then fitted into triangular meshes (e), and the displacement of each node on the mesh between consecutive acquisition time points was tracked to estimate the stress field that caused deformation (e). The calculation shows that the stress varies temporally and spatially, ranging from $10 \mathrm{nN} / \mu \mathrm{m}^{2}$ to $30 \mathrm{nN} / \mu \mathrm{m}^{2}$, resulting in heterogeneous deformation (e). The correlation coefficient between the deformation and cell density is low $(\mathrm{R} 2=0.0255, \mathrm{f})$. The time stamp shown in each image indicates the time when the image was acquired after cell encapsulation. Scale bars: $1 \mathrm{~mm}$ in (a)(b)(c) (d) and (e). 
and green, respectively. By visual inspection, the deformation and the cell cluster distribution appeared to have a weak correlation as the coefficient between deformation and cell density was low $\left(\mathrm{R}^{2}=\right.$ 0.0255 , figure $3 \mathrm{f}$ ), implying that the cell clusters did not act as a synchronized population. It is possible that a portion of the clusters did not contribute to force generation ${ }^{49}$, or that forces generated in opposite directions by different clusters canceled each other, resulting in little deformation in some high cell-density areas.

Collective cell motility and cellular mechanical force tracked by timelapse MRI. To more precisely track collective force generation by a single cluster, we constructed a 3D ECM system where a single MDCK cell cluster and numerous $50-\mu \mathrm{m}$ diameter polystyrene beads, serving as fiduciary markers, were embedded in a $10 \mathrm{~mm} \times$ $5 \mathrm{~mm} \times 1 \mathrm{~mm}$ collagen gel. The single MDCK cell cluster (figure 4a) was pre-formed by a hanging drop protocol, embedded into the collagen gel, and then scanned for 30 hours using timelapse MRI (figure $4 \mathrm{~b}$, movie S5). The cluster was segmented in the timelapsed images by its size and $\mathrm{T}_{2}{ }^{*}$ contrast. Tracking of the segmented cluster showed it migrated at an average rate of $0.25 \mu \mathrm{m} / \mathrm{min}$, slowly but persistently moving in a consistent direction throughout the imaging period (figure $4 \mathrm{~b}$ ). The local displacements of the embedded fiduciary markers were tracked by particle image velocimetry (PIV) and a finite element mesh was constructed, where node positions were assigned based on the fiduciary marker locations in the first frame of the timelapse image. The node displacements were then assigned with the displacements calculated by PIV between two consecutive time points. The stress field was reconstructed based on the node displacement using formulas (1) and (2). The results showed the stress ranged from $2 \mathrm{nN} / \mu \mathrm{m}^{2}$ to $20 \mathrm{nN} / \mu \mathrm{m}^{2}$, and the stress amplitude dropped gradually with the distance from the MDCK cell cluster (figure 4d).

Two types of force generation have been reported in collective cell migration: (1) "pulling" forces mediated by adhesion-complexes that connect to the actin cytoskeleton ${ }^{2,50,51}$ at the trailing end of a moving cell aggregate; and, (2) "pushing" forces, which are produced by a stable protrusion formed by multiple cells at the leading edge of a moving cell aggregat ${ }^{52,53}$. Pulling forces have been widely reported in collective migration of mesenchymal cells, epithelial cells, and cancer invasion $^{2,50,51}$. Pushing forces, however, are less well understood and yet to be extensively studied. By tracking the local displacement of the fiduciary markers, we found that the cell cluster generated both pulling and pushing forces at the trailing end and leading edge relative to the moving direction, respectively. We also found that the fiduciary markers sometimes moved locally in a rotating manner (figure $4 \mathrm{c}$ at time 7:00 and 20:00), indicating the cell clusters also rotated while migrating in addition to translating laterally, which is consistent with previous microscopic observations ${ }^{54}$.

MRI-based approaches to track force generation and collective cell motility applied to additional cell lines. To demonstrate that MRI contrast can be utilized in the detection of aggregated cells other than MDCKs, human bladder carcinoma cells (NBT-2) were seeded in 3D collagen and allowed to form clusters as described previously, before imaging. NBT-2 cell clusters were detected both in MRI (figure 5a) and optical microscopy (figure 5b). Furthermore, a 3D collagen gel containing one single pre-formed cluster of NBT-2 cells and $50-\mu \mathrm{m}$ diameter polystyrene beads was imaged for 5 hours and, based on the displacement of the embedded polystyrene beads in consecutive frames, a stress field was generated (figure $5 \mathrm{e}$, movie S8). We observed similar pulling, pushing and rotating forces as compared to the MDCK cells (figure 5f, movie S9).

In addition to the NBT-2 cells, a $3 \mathrm{D}$ collagen gel containing mouse embryonic fibroblast (MEF) cells was imaged by MRI. Eight $\times 10^{7}$ MEF cells were pre-labeled with $30-\mathrm{nm}$ iron oxide particles to enhance the contrast. Deformation of the 3D ECM and cell migration within 10 hours were detected, though by visual inspection the deformation process was notably faster (figure 5c, movie S6) compared to MDCK cells. The estimated stress field based on the deformation of the collagen gel confirms that higher stress was indeed generated by MEF cells (figure 5d, movie S7).

\section{Discussion}

In the present study we demonstrated that MRI is a useful tool to investigate cell mechanics at a mesoscopic scale, permitting imaging of cells embedded in thick 3D ECM, a challenge when using conventional optical microscopy. Additionally, we demonstrated that MRI enables simultaneous monitoring of dynamic cell distribution and ECM deformation/remodeling. Furthermore, tracking the local displacement of embedded fiduciary markers allowed the estimation of the dynamic stress field generated by the cell clusters, showing that MRI is a versatile tool for imaging cell-ECM interactions.

Imaging cells by MRI opens up the possibility of more systematic tracking of cells and the mechanical forces they generate in $3 \mathrm{D}$, especially in complex matrices that exceed $0.5 \mathrm{~mm}$ in thickness. Currently most cell mechanics studies are performed in $2 \mathrm{D}$ culture or very thin $3 \mathrm{D}$ film $(<500 \mu \mathrm{m})$ because of the field depth restriction imposed by optical microscopy. Given that the behavior and morphology of the cells are drastically different in $2 \mathrm{D}$ and $3 \mathrm{D}$ contexts ${ }^{55-58}$, and that $3 \mathrm{D}$ ECM is more representative of physiological conditions, 3D imaging by MRI may reveal critical insights into cell behaviors that are not readily evident by optical microscopy.

The $\mathrm{T}_{2}{ }^{*}$ contrast is most likely generated because MDCK/NBT-2 cells cultured in DMEM (ingredients include ferric nitrate) contain sufficient endogenous iron for a small cluster to be detected by MRI. MDCK is a common cell line model for studying collective migration $^{30,33,59}$, tubulogenesis ${ }^{32,60,61}$, and tight junctions ${ }^{62-64}$, and has been well characterized at a microscopic level. Visualization by MRI can now extend the study of this well-established model to multiple size scales, owing in part to the fact that unlabeled MDCK cells can be visualized. However, if the MRI imaging technique used here is to be widely applied to additional cell lines in the future, supplements such as iron (III) nitrate nonahydrate may be required to generate the needed contrast. Alternatively, cells with undetectable MR contrast such as MEF cells could be labeled with iron oxide particles (figure $4 \mathrm{c})^{24,26,65}$, which can also be used to track single cells ${ }^{24}$ as well as cell clusters.

The pre-formed cell cluster migrated slowly in the polystyreneembedded 3D ECM (figure 4b) compared to the cell clusters formed naturally from single cells within the ECM (figure 1d). The difference indicates that cell motility changes over time after cells form clusters and is consistent with the prior observation that pre-formed epithelial acini exhibit a slow migration speed ${ }^{9}$. This observation may also be caused by physical constraints due to the relatively large size of the clusters $^{34}$. Despite the slower motility of the pre-formed cluster (figure 4), we found that pre-clustered cells exerted detectable pushing, pulling and rotating forces onto the environment. The constant exertion may be an element in the ECM remodeling processes as previous reports have shown that ECM containing highly contractile cells undergoes vigorous remodeling ${ }^{44}$. Looking forward, it will be interesting to use MRI to test if various experimental manipulations, such as perturbation of proteins known to be involved in force generation (myosin, actin, microtubules, and associated regulatory proteins), alter the amplitude or direction of these forces.

It is worth noting that recording collective cell behavior at mesoscopic scales usually requires imaging the specimen for hours to days ${ }^{27,29}$, during which time cell division can occur and increase the total cell number contained in the matrix. Therefore, changes in the stress field over time should be interpreted as a combination of both increased contractility in single cells and increased cell number. Since cell division s occur also in aggregated cells in vivo, the observation of these changes from an in vitro system like ours should not 

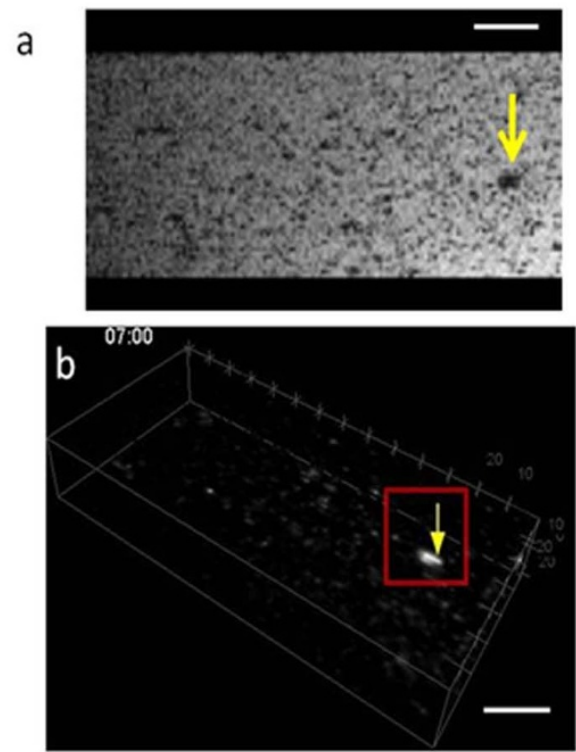

C
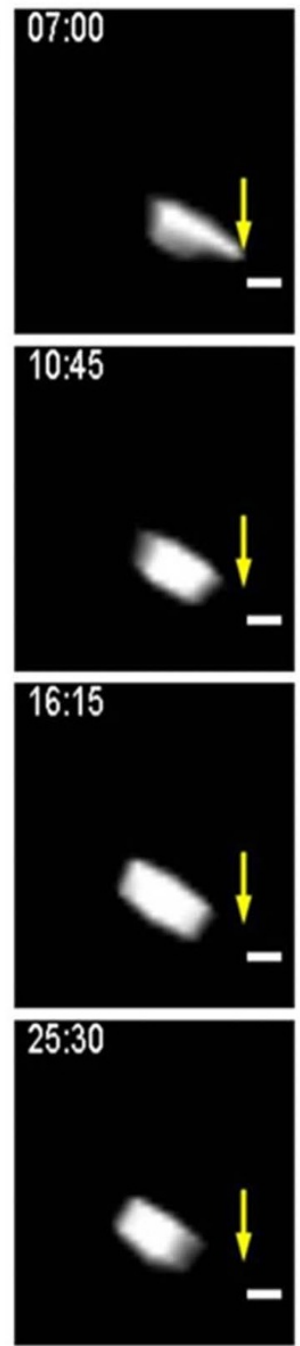

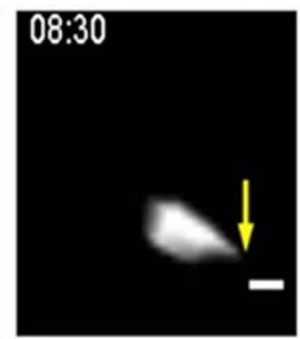

\section{4:00}
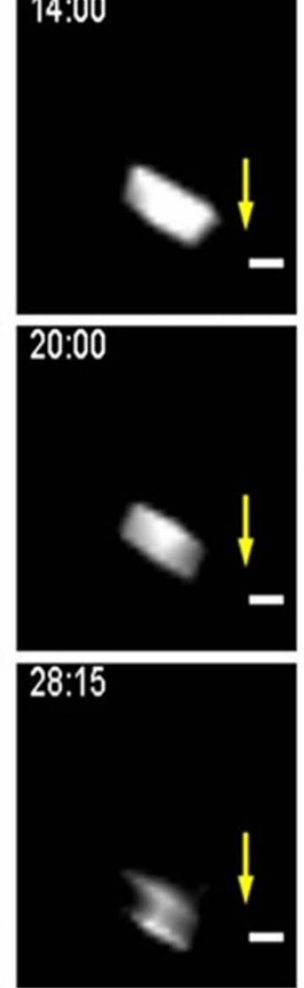
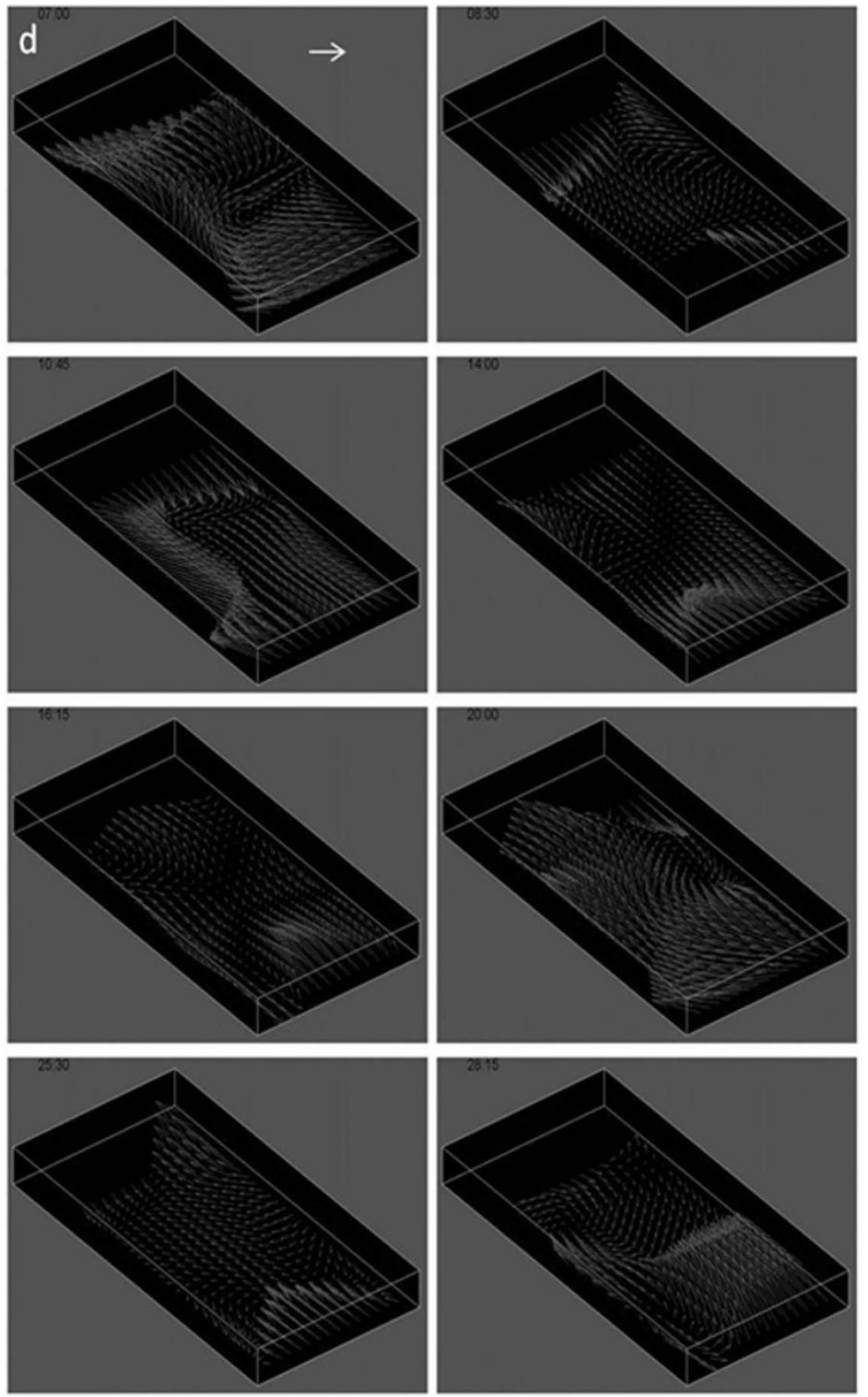

e

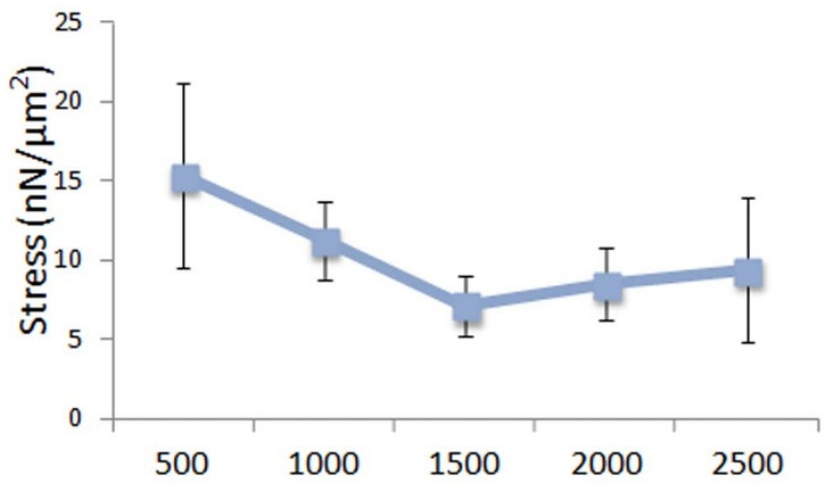

Distance from the cell cluster $(\mu \mathrm{m})$

Figure $4 \mid$ Collective migration and cellular mechanical force tracked by timelapse MRI. A pre-formed cell cluster and numerous $50-\mu \mathrm{m}$ polystyrene beads as fiduciary markers were embedded in 3D collagen gel. The collagen gel was scanned for 30 hours for timelapse acquisition. The 3D-projected MRI image at the beginning of the timelapse series is shown in (a). The inverted 3D reconstructed MRI image at the beginning of the timelapse series is shown in (b). The volume highlighted by red box in (b) at different time points was shown in (c) where the cell cluster (indicated by the yellow arrow in a, b and c) moved at the average rate of $0.25 \mu \mathrm{m} / \mathrm{min}$. The stress field caused by the force-exerting cell cluster was calculated based on the displacement of polystyrene beads; the amplitude and orientation of the stress at each spatial location over the time is expressed in vector form (d). The yellow arrow indicates the location of the cell cluster. The length of the white arrow represents $10 \mathrm{nN} / \mu \mathrm{m}^{2}$. The average stress, from three independent experiments, slowly dissipates as the distance from the cells increases (e). The time stamp shown in each image indicates the time when the image was acquired after cell encapsulation in the collagen gel. Scale bars: $1 \mathrm{~mm}$ in (a), (b); $200 \mu \mathrm{m}$ in (c). 


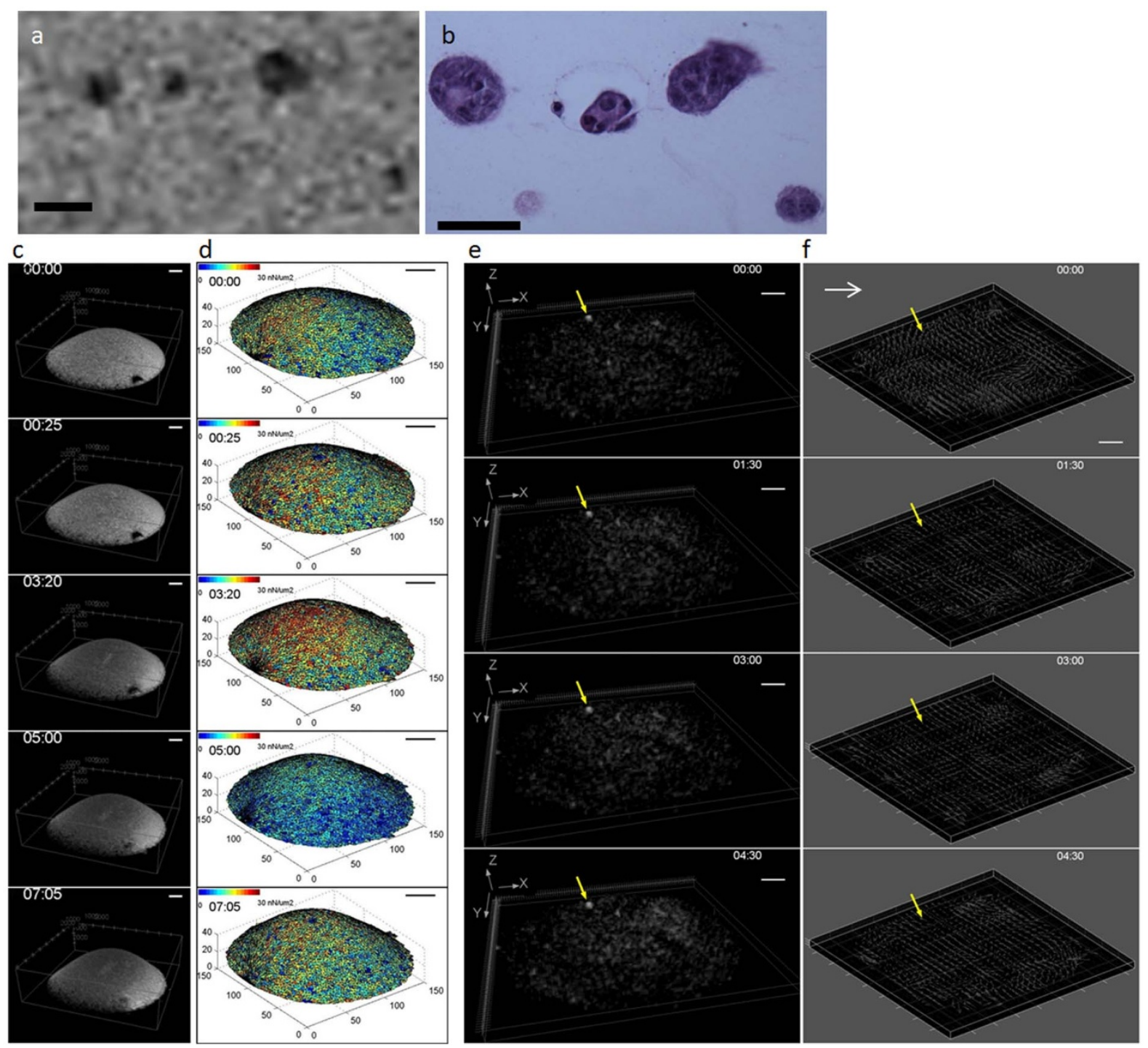

Figure $5 \mid$ MRI-based approaches to track force generation and collective cell motility can be generally applied to other cell lines. MRI contrast can also be detected in clustered Rat bladder carcinoma NBT-2 cells (a), which corresponds to the H\&E staining of the same sample by optical microscopy (b). $8 \times 10^{7} \mathrm{MEFs}$ pre-labeled with 30-nm iron oxide particles for contrast enhancement and embedded in collagen gel. The deformation of the 3D ECM and cell migration within 10 hours were detected (c). The estimated stress field based on the deformation of the collagen gel shows that higher stress was generated by MEF cells (d) compared to MDCK cells (see figure 3). 3D collagen gel containing one single pre-formed cluster of NBT-2 cells and $50-\mu \mathrm{m}$ diameter polystyrene beads was imaged for 5 hours (e). Based on displacement of the embedded polystyrene beads in consecutive frames, the stress field was generated (f). The time stamp shown in each image indicates the time when the image was taken relative to cell encapsulation. The length of the white arrow represents $10 \mathrm{nN} / \mu \mathrm{m}^{2}$. Scale bars: $1 \mathrm{~mm}$.

necessarily be regarded as artifacts. However, no attempt has been made to assign cell numbers to the clusters. A quantitative study exploring the relationship between $\mathrm{T}_{2}{ }^{*}$ values and cell numbers might allow us to dissect the different factors (cell number and contractility) contributing to the changes in force generation.

In summary, we established an MRI-based platform that enables simultaneous observation of ECM deformation/remodeling and dynamic cell distribution at mesoscopic scales. This platform may be a useful tool in efforts to develop more effective cancer treatments, or for designing artificial organogenesis systems, where migrationassociated ECM deformation/modeling can be recorded when embedded cells are targeted with perturbation of specific proteins. It is believed that invasive tumors are facilitated by ECM deformation and remodeling ${ }^{66}$ and that ECM remodeling might result in low drug delivery $^{67}$. The MRI platform presented here can be utilized to quantitatively and qualitatively assess this process to better understand how ECM deformation/modeling can affect drug distribution into and throughout tumors.

\section{Methods}

Cell culture. MDCK, NBT-2 and MEF cells were acquired from ATCC. All cells were cultured in Dulbecco's modified Eagle medium (DMEM) supplemented with 10\% fetal bovine serum (FBS), $5 \mathrm{mg} / \mathrm{ml}$ penicillin, and $5 \mathrm{mg} / \mathrm{ml}$ streptomycin (Invitrogen Inc.). Cells were maintained at $37^{\circ} \mathrm{C}$ under a humidified incubator with $5 \%$ carbon dioxide. The culture medium was changed every 2 days. Before every experiment, cells were detached from the culture flask by $0.25 \%$ trypsin-EDTA (Invitrogen). The cell suspension was then centrifuged at $1200 \mathrm{rpm}$ for $5 \mathrm{~min}$ and the cell pellets were re-suspended in DMEM medium.

GFP transfection was performed using Amaxa Nucleofector electroporator (Lonza). Briefly, in each preparation, $10^{6} \mathrm{MDCK}$ cells were co-incubated with $3 \mu \mathrm{g}$ of EGFP cDNA in $100 \mu \mathrm{l}$ of Nucleofector Solution using the program A-024.

MEF cells were labeled for MR contrast by the following steps: $1 \mu \mathrm{l}$ of $30-\mathrm{nm}$ iron oxide particles suspension in water (Ocean NanoTech) was added to $10^{6}$ MEF cells and incubated for 2 hours, followed by washing 3 times with PBS. Labeled MEF cells 
were then cultured in DMEM for another hour before being subjected trypsinization and other experimental procedures.

HGF treatment was performed by adding HGF into the collagen mixture at the time of cell embedding (see below) to achieve a final concentration of $10 \mathrm{ng} / \mathrm{ml}$.

Pre-formed cell cluster. The pre-formed MDCK or NBT-2 cell clusters were generated by modified protocols as previously described ${ }^{68} .20 \mu \mathrm{l}$ of resuspended cells $\left(2 \times 10^{4}\right)$ were deposited on the underside of the lid of a $10-\mathrm{cm}$ tissue culture dish. The bottom of the dish contained 5-ml of PBS and served to prevent evaporation of the drops by forming a hydration chamber. Inverting the lid over the hydration chamber created hanging drops. The drops were then incubated at $37^{\circ} \mathrm{C}, 5 \% \mathrm{CO}_{2}$, and $95 \%$ humidity for 12 hours to allow the cells to coalesce into clusters before seeding them onto neutralized 3D collagen in liquid form prior to gelation.

Cells encapsulation by 3D collagen gel. Collagen type I gels were prepared by mixing appropriate volumes of collagen solution (Gibco) with $5 \times$ DMEM (Invitrogen), $1 \%$ HEPES buffer (Gibco) and $1 \mathrm{~N} \mathrm{NaOH}$ with pre-cooled pipet tips in pre-cooled eppendorf tubes to produce a final collagen concentration of $2 \mathrm{mg} / \mathrm{ml}$. Resuspended cells or pre-formed clusters were then added to the cold collagen while still in liquid form. After the addition of the cells the mixture was kept at $37^{\circ} \mathrm{C}$ for 30 minutes to enable complete gelation.

MRI acquisition and image analysis. The timelapse MRI of cell-embedded matrices was performed in $11.7 \mathrm{~T}, 30 \mathrm{~cm}$ magnet (Magnex, Oxford) interfaced to a small animal MRI scanner (Bruker). The cradle of the scanner was equipped with tubing connected to a $37^{\circ} \mathrm{C}$ water bath to keep the cell-containing samples at a viable temperature. Spin echo sequence was used to acquire 3D time series images $\left(\mathrm{TE} / \mathrm{TR}=5 / 30 \mathrm{~ms}\right.$, flip angle $\left.=15^{\circ}\right)$ with an isotropic resolution of $50 \mu \mathrm{m} \times 50 \mu \mathrm{m}$ $\times 50 \mu \mathrm{m} / \mathrm{voxel}$. Time intervals between consecutive acquisitions and the total imaging period were indicated in the individual experimental descriptions in the Results section. $\mathrm{T}_{2}$ and $\mathrm{T}_{2}{ }^{*}$ measurements were performed using spin echo $(\mathrm{TE} / \mathrm{TR}=6 / 1500 \mathrm{~ms}, 12$ echoes with linear inter-echo spacing) and gradient echo (TE/TR $=4 / 1500 \mathrm{~ms}, 12$ echoes with linear inter-echo spacing) sequences, respectively. After imaging, the samples were fixed for histological analysis as described below.

MRI images were processed in ImageJ software (NIH) and the segmentation was performed by thresholding with size and grayscale criteria. Tracking migrating cell clusters in inverted MRI images was performed using the Mosaic plugin of ImageJ (available at http://www.mosaic.ethz.ch/Downloads/ParticleTracker). $\mathrm{T}_{2}$ and $\mathrm{T}_{2}{ }^{*}$ relaxation times were exponentially fitted by CurveFitting plugin of ImageJ.

Microscopy. Fluorescent images were acquired on an Olympus X71 microscope equipped with a CCD-camera (Hamamatsu). A Zeiss Axiovert microscope equipped with a color camera was used to examine histochemical staining.

Histological studies of cell-embedded 3D matrices. The cell-embedded collagen gels were first processed into formalin-fixed paraffin-embedded (FFPE) blocks as follows: The cell embedded ECM gels were fixed in $10 \%$ neutral buffered formalin overnight at room temperature and embedded into paraffin wax. 5 - $\mu \mathrm{m}$ thick sections were then cut from the FPPE blocks and placed onto histology glass slides for further processing.

To prepare for staining, slides were loaded into glass slide holders and deparaffinized in solutions in the following order: Twice in $100 \%$ xylenes for 5 minutes, once in $100 \%$ ethanol for 5 minutes, once in $90 \%$ ethanol for 5 minutes, once in $70 \%$ ethanol for 5 minutes, and once in $\mathrm{ddH}_{2} \mathrm{O}$ for 5 minutes. The slides were then stained with hematoxylin and eosin (H\&E), or for iron content using a staining kit (Scientific Device Laboratory, Inc.).

Particle displacement analysis. Acquired MRI Images were first inverted into darkbackground images. The cell cluster in the images was excluded by size thresholding segmentation, producing images in which only polystyrene beads were present. Displacement fields were then calculated using particle imaging velocimetry (PIV) software in Matlab (available at http://www.oceanwave.jp/softwares/mpiv/), using the minimum quadratic differences (MQD) algorithm that calculates the shift necessary to produce the minimum cross-correlation coefficient between a small region of the experimental image and the reference image. The software uses recursively computed displacement in a small grid spacing using information from the previous computations to filter false vectors caused by noise. Displacement vectors were filtered and interpolated using the Kriging interpolation method. To evaluate 3D displacement, the XY planes of the images were first computed followed by the calculation of XZ planes. The $3 \mathrm{D}$ displacement vectors were then reconstructed by imposition.

Mesh generation. 3D time series images were fitted to triangular meshes with fixed numbers of nodes (1340) using modified Matlab codes from ISO2MESH (available at http://iso2mesh.sourceforge.net/cgi-bin/index.cgi?Home).

Stress field estimation. Mechanical stress was calculated as a product of the stiffness matrix with the node displacements following formulas (1) and (2) described in the Results section. Computing was performed using programs written in Matlab (Mathworks).
1. Montell, D. J. Morphogenetic Cell Movements: Diversity from Modular Mechanical Properties. Science 322, 1502-1505 (2008).

2. Friedl, P. \& Gilmour, D. Collective cell migration in morphogenesis, regeneration and cancer. Nat Rev Mol Cell Biol 10, 445-457 (2009).

3. Hegerfeldt, Y., Tusch, M., Brocker, E.-B. \& Friedl, P. Collective Cell Movement in Primary Melanoma Explants. Cancer Research 62, 2125-2130 (2002).

4. Wolf, K. et al. Multi-step pericellular proteolysis controls the transition from individual to collective cancer cell invasion. Nat Cell Biol 9, 893-904 (2007).

5. Weber Gregory, F., Bjerke Maureen, A. \& DeSimone Douglas, W. A Mechanoresponsive Cadherin-Keratin Complex Directs Polarized Protrusive Behavior and Collective Cell Migration. Developmental Cell 22, 104-115 (2011).

6. Tomasek, J. J., Gabbiani, G., Hinz, B., Chaponnier, C. \& Brown, R. A. Myofibroblasts and mechano-regulation of connective tissue remodelling. Nat Rev Mol Cell Biol 3, 349-363 (2002).

7. Legant, W. R. et al. Microfabricated tissue gauges to measure and manipulate forces from 3D microtissues. Proceedings of the National Academy of Sciences 106, 10097-10102 (2009).

8. Cukierman, E. \& Bassi, D. E. Physico-mechanical aspects of extracellular matrix influences on tumorigenic behaviors. Semin Cancer Biol 20, 139-145 (2010).

9. Pearson, G. W. \& Hunter, T. Real-time imaging reveals that noninvasive mammary epithelial acini can contain motile cells. The Journal of Cell Biology 179, 1555-1567 (2007).

10. Debnath, J., Muthuswamy, S. K. \& Brugge, J. S. Morphogenesis and oncogenesis of MCF-10A mammary epithelial acini grown in three-dimensional basement membrane cultures. Methods 30, 256-268 (2003).

11. Zhao, L., Lee, V. K., Yoo, S. S., Dai, G. \& Intes, X. The integration of 3-D cell printing and mesoscopic fluorescence molecular tomography of vascular constructs within thick hydrogel scaffolds. Biomaterials 33, 5325-5332 (2012).

12. Jiang, Y., Tong, Y. \& Lu, S. Visualizing the three-dimensional mesoscopic structure of dermal tissues. J Tissue Eng Regen Med (2012).

13. Bian, W., Liau, B., Badie, N. \& Bursac, N. Mesoscopic hydrogel molding to control the 3D geometry of bioartificial muscle tissues. Nat Protoc 4, 1522-1534 (2009).

14. Fidler, I. J. The pathogenesis of cancer metastasis: the 'seed and soil' hypothesis revisited. Nat Rev Cancer 3, 453-458 (2003).

15. Ji, N., Shroff, H., Zhong, H. \& Betzig, E. Advances in the speed and resolution of light microscopy. Current Opinion in Neurobiology 18, 605-616 (2008).

16. Huisken, J., Swoger, J., Del Bene, F., Wittbrodt, J. \& Stelzer, E. H. K. Optical Sectioning Deep Inside Live Embryos by Selective Plane Illumination Microscopy. Science 305, 1007-1009 (2004).

17. Wu, Y. et al. Inverted selective plane illumination microscopy (iSPIM) enables coupled cell identity lineaging and neurodevelopmental imaging in Caenorhabditis elegans. Proceedings of the National Academy of Sciences 108, 17708-17713 (2011).

18. Amnon, B., Karthikan, R., Andre, E. X. B. \& Dennis, E. D. How deeply cells feel: methods for thin gels. Journal of Physics: Condensed Matter 22, 194116 (2010).

19. Modo, M. et al. Tracking Transplanted Stem Cell Migration Using Bifunctional, Contrast Agent-Enhanced, Magnetic Resonance Imaging. NeuroImage 17, 803-811 (2002).

20. Guzman, R. et al. Long-term monitoring of transplanted human neural stem cells in developmental and pathological contexts with MRI. Proceedings of the National Academy of Sciences 104, 10211-10216 (2007).

21. Ye, Q. et al. Longitudinal tracking of recipient macrophages in a rat chronic cardiac allograft rejection model with noninvasive magnetic resonance imaging using micrometer-sized paramagnetic iron oxide particles. Circulation 118 , 149-156 (2008).

22. Sumner, J. P., Shapiro, E. M., Maric, D., Conroy, R. \& Koretsky, A. P. In vivo labeling of adult neural progenitors for MRI with micron sized particles of iron oxide: quantification of labeled cell phenotype. Neuroimage 44, 671-678 (2009)

23. Townson, J. L. et al. Three-Dimensional Imaging and Quantification of Both Solitary Cells and Metastases in Whole Mouse Liver by Magnetic Resonance Imaging. Cancer Research 69, 8326-8331 (2009).

24. Shapiro, E. M., Sharer, K., Skrtic, S. \& Koretsky, A. P. In vivo detection of single cells by MRI. Magn Reson Med 55, 242-249 (2006).

25. Huang, S. et al. Using magnetic resonance microscopy to study the growth dynamics of a glioma spheroid in collagen I: A case study. BMC Medical Imaging 8, 3 (2008).

26. Kruttwig, K. et al. Development of a three-dimensional in vitro model for longitudinal observation of cell behavior: monitoring by magnetic resonance imaging and optical imaging. Mol Imaging Biol 12, 367-376 (2010).

27. Boudou, T. et al. A microfabricated platform to measure and manipulate the mechanics of engineered cardiac microtissues. Tissue engineering 18, 910-919 (2011).

28. Ilagan, R. et al. Linear measurement of cell contraction in a capillary collagen gel system. Biotechniques 48, 153-155 (2010).

29. Vernon, R. B. \& Gooden, M. D. An improved method for the collagen gel contraction assay. In Vitro Cell Dev Biol Anim 38, 97-101 (2002).

30. Arciero, J. C., Mi, Q., Branca, M. F., Hackam, D. J. \& Swigon, D. Continuum model of collective cell migration in wound healing and colony expansion. Biophys J 100, 535-543 (2011).

31. du Roure, O. et al. Force mapping in epithelial cell migration. Proceedings of the National Academy of Sciences of the United States of America 102, 2390-2395 (2005). 
32. Kang, T. et al. Functional characterization of MT3-MMP in transfected MDCK cells: progelatinase A activation and tubulogenesis in 3-D collagen lattice. The FASEB Journal 14, 2559-2568 (2000).

33. Poujade, M. et al. Collective migration of an epithelial monolayer in response to a model wound. Proceedings of the National Academy of Sciences 104, 15988-15993 (2007)

34. Puliafito, A. et al. Collective and single cell behavior in epithelial contact inhibition. Proceedings of the National Academy of Sciences 109, 739-744 (2012).

35. Wood, J. C. et al. Relationship between labile plasma iron, liver iron concentration and cardiac response in a deferasirox monotherapy trial. Haematologica 96, 1055-1058 (2011)

36. Wu, G., Xi, G., Hua, Y. \& Sagher, O. T2* Magnetic Resonance Imaging Sequences Reflect Brain Tissue Iron Deposition Following Intracerebral Hemorrhage. Transl Stroke Res 1, 31-34 (2010).

37. Hansen, B. \& Vestergaard-Poulsen, P. Mapping the parameter space of a T2dependent model of water diffusion MR in brain tissue. Magn Reson Imaging 24, 1031-1038 (2006).

38. Righini, A., Ramenghi, L. A., Parini, R., Triulzi, F. \& Mosca, F. Water apparent diffusion coefficient and T2 changes in the acute stage of maple syrup urine disease: evidence of intramyelinic and vasogenic-interstitial edema. J Neuroimaging 13, 162-165 (2003).

39. Wellen, J., Helmer, K. G., Grigg, P. \& Sotak, C. H. Spatial characterization of T1 and $\mathrm{T} 2$ relaxation times and the water apparent diffusion coefficient in rabbit Achilles tendon subjected to tensile loading. Magn Reson Med 53, 535-544 (2005).

40. Wu, J. C. et al. In vivo determination of the anisotropic diffusion of water and the $\mathrm{T} 1$ and T2 times in the rabbit lens by high-resolution magnetic resonance imaging. Invest Ophthalmol Vis Sci 34, 2151-2158 (1993).

41. Grotegut, S., von Schweinitz, D., Christofori, G. \& Lehembre, F. Hepatocyte growth factor induces cell scattering through MAPK/Egr-1-mediated upregulation of Snail. EMBO J 25, 3534-3545 (2006).

42. Ridley, A. J., Comoglio, P. M. \& Hall, A. Regulation of scatter factor/hepatocyte growth factor responses by Ras, Rac, and Rho in MDCK cells. Molecular and Cellular Biology 15, 1110-1122 (1995).

43. Weidner, K. M., Sachs, M. \& Birchmeier, W. The Met receptor tyrosine kinase transduces motility, proliferation, and morphogenic signals of scatter factor/ hepatocyte growth factor in epithelial cells. The Journal of Cell Biology 121, 145-154 (1993).

44. Goetz Jacky, G. et al. Biomechanical Remodeling of the Microenvironment by Stromal Caveolin-1 Favors Tumor Invasion and Metastasis. Cell 146, 148-163 (2011).

45. Ngo, P., Ramalingam, P., Phillips, J. A. \& Furuta, G. T. in Cell-Cell Interactions. Vol. 341, 103-1092006.

46. Roeder, B. A., Kokini, K., Sturgis, J. E., Robinson, J. P. \& Voytik-Harbin, S. L. Tensile mechanical properties of three-dimensional type I collagen extracellular matrices with varied microstructure. Journal of Biomechanical Engineering 124, 214-222 (2002).

47. Stein, A. M., Vader, D. A., Weitz, D. A. \& Sander, L. M. The micromechanics of three-dimensional collagen-I gels. Complexity 16, 22-28 (2011).

48. Koch, T. M., Münster, S., Bonakdar, N., Butler, J. P. \& Fabry, B. 3D Traction Forces in Cancer Cell Invasion. PLoS ONE 7, e33476 (2012).

49. Puliafito, A. et al. Collective and single cell behavior in epithelial contact inhibition. Proceedings of the National Academy of Sciences 109, 739-744 (2012).

50. Hegerfeldt, Y., Tusch, M., Brocker, E.-B. \& Friedl, P. Collective Cell Movement in Primary Melanoma Explants. Cancer Research 62, 2125-2130 (2002).

51. Trepat, X. et al. Physical forces during collective cell migration. Nat Phys 5, 426-430 (2009).

52. Ewald, A. J., Brenot, A., Duong, M., Chan, B. S. \& Werb, Z. Collective epithelial migration and cell rearrangements drive mammary branching morphogenesis. Dev Cell 14, 570-581 (2008).

53. Schmidt, S. \& Friedl, P. Interstitial cell migration: integrin-dependent and alternative adhesion mechanisms. Cell Tissue Res 339, 83-92 (2009).

54. Marmaras, A. et al. A mathematical method for the 3D analysis of rotating deformable systems applied on lumen-forming MDCK cell aggregates. Cytoskeleton 67, 224-240 (2010).
55. Doyle, A. D., Wang, F. W., Matsumoto, K. \& Yamada, K. M. One-dimensional topography underlies three-dimensional fibrillar cell migration. The Journal of Cell Biology 184, 481-490 (2009).

56. Fraley, S. I. et al. A distinctive role for focal adhesion proteins in three-dimensional cell motility. Nat Cell Biol 12, 598-604 (2010).

57. Zaman, M. H. et al. Migration of tumor cells in 3D matrices is governed by matrix stiffness along with cell-matrix adhesion and proteolysis. Proceedings of the National Academy of Sciences 103, 10889-10894 (2006).

58. Yamazaki, D., Kurisu, S. \& Takenawa, T. Involvement of Rac and Rho signaling in cancer cell motility in 3D substrates. Oncogene 28, 1570-1583 (2009).

59. Tambe, D. T. et al. Collective cell guidance by cooperative intercellular forces. Nat Mater 10, 469-475 (2011).

60. Hellman, N. E. et al. Activated extracellular signal-regulated kinases are necessary and sufficient to initiate tubulogenesis in renal tubular MDCK strain I cell cysts. American Journal of Physiology - Renal Physiology 289, F777-F785 (2005).

61. Popsueva, A. et al. GDNF promotes tubulogenesis of GFRÎ \pm 1 -expressing MDCK cells by Src-mediated phosphorylation of Met receptor tyrosine kinase. The Journal of Cell Biology 161, 119-129 (2003).

62. Kovbasnjuk, O., Leader, J. P., Weinstein, A. M. \& Spring, K. R. Water does not flow across the tight junctions of MDCK cell epithelium. Proceedings of the National Academy of Sciences 95, 6526-6530 (1998).

63. Muresan, Z., Paul, D. L. \& Goodenough, D. A. Occludin 1B, a Variant of the Tight Junction Protein Occludin. Molecular Biology of the Cell 11, 627-634 (2000).

64. Zheng, B. \& Cantley, L. C. Regulation of epithelial tight junction assembly and disassembly by AMP-activated protein kinase. Proceedings of the National Academy of Sciences 104, 819-822 (2007).

65. Wu, Y. L. et al. In situ labeling of immune cells with iron oxide particles: An approach to detect organ rejection by cellular MRI. Proceedings of the National Academy of Sciences of the United States of America 103, 1852-1857 (2006).

66. Wyckoff, J. B., Pinner, S. E., Gschmeissner, S., Condeelis, J. S. \& Sahai, E. ROCKand Myosin-Dependent Matrix Deformation Enables Protease-Independent Tumor-Cell Invasion In Vivo. Current Biology 16, 1515-1523 (2006).

67. Netti, P. A., Berk, D. A., Swartz, M. A., Grodzinsky, A. J. \& Jain, R. K. Role of extracellular matrix assembly in interstitial transport in solid tumors. Cancer Res 60, 2497-2503 (2000)

68. Thoreson, M. A. \& Reynolds, A. B. Altered expression of the catenin p120 in human cancer: implications for tumor progression. Differentiation 70, 583-589 (2002).

\section{Acknowledgements}

This research was supported (in part) by the Intramural Research Program of the NIH, NINDS, and also the Center for Cancer Research, NCI.

\section{Author contributions}

Y.C. designed and executed the experiments and wrote the paper. S.J.D. contributed to MRI acquisition. M.A.T. contributed to the histology studies and writing. M.R.E.-B. contributed to histology studies and writing. A.K.P. contributed to critical discussions of the experiment design

\section{Additional information}

Supplementary information accompanies this paper at http://www.nature.com/ scientificreports

Competing financial interests: The authors declare no competing financial interests.

License: This work is licensed under a Creative Commons

Attribution-NonCommercial-NoDerivs 3.0 Unported License. To view a copy of this license, visit http://creativecommons.org/licenses/by-nc-nd/3.0/

How to cite this article: Chen, Y., Dodd, S.J., Tangrea, M.A., Emmert-Buck, M.R. \& Koretsky, A.P. Measuring collective cell movement and extracellular matrix interactions using magnetic resonance imaging. Sci. Rep. 3, 1879; DOI:10.1038/srep01879 (2013) 